\title{
Evaluations of some physical properties for oil palm as alternative biomass resources
}

\begin{abstract}
The objective of this study was to investigate the moisture content (MC), density, and amount of extractives along the height of a 32-year-old oil palm stem. The extractives were removed following TAPPI standards T-207 and T-280 for water solubility and acetone extractives. The results showed that the $\mathrm{MC}$ of the palm stem increased from the outer towards the inner section, while the density decreased. Along the tree height, the MC was found to increase from the bottom to the middle part, but slightly decreased towards the top. An inverse trend was obtained for the density distribution along the tree height. The results of the extractive separation showed that the middle and center sections of the oil palm stem contained the highest amount of extractives irrespective of the types of solvent. The highest amount of extractives was obtained from hot water extraction, followed by cold water and acetone extractions. The lowest amount of extractives was located at the bottom outer section of the oil palm stem which ranging from 2.0 to $9.2 \%$, whereas the middle and center sections contained a greater amount of extractives ranged from 4.6 to $32.8 \%$ regardless of the type of solvent used.
\end{abstract}

Keyword: Density; Extractive; Moisture content; Oil palm stem; Parenchyma; Vascular bundles 